Revue des patrimoines

$28 \mid 2016$

Le moulage. Pratiques historiques et regards contemporains

\title{
Mouler, tirer, modifier, mouler à nouveau. Rodin et le moulage
}

Rodin and casting.

\section{François Blanchetière}

\section{(2) OpenEdition}

Journals

Édition électronique

URL : https://journals.openedition.org/insitu/12743

DOI : $10.4000 /$ insitu. 12743

ISSN : 1630-7305

Éditeur

Ministère de la Culture

Référence électronique

François Blanchetière, "Mouler, tirer, modifier, mouler à nouveau. Rodin et le moulage », In Situ [En

ligne], 28 | 2016, mis en ligne le 15 mars 2016, consulté le 21 septembre 2021. URL : http://

journals.openedition.org/insitu/12743; DOI : https://doi.org/10.4000/insitu.12743

Ce document a été généré automatiquement le 21 septembre 2021.

\section{cc) () $९$}

In Situ Revues des patrimoines est mis à disposition selon les termes de la licence Creative Commons Attribution - Pas d'Utilisation Commerciale - Pas de Modification 4.0 International. 


\title{
Mouler, tirer, modifier, mouler à nouveau. Rodin et le moulage
}

\author{
Rodin and casting.
}

\author{
François Blanchetière
}

Communication présentée lors des journées d'étude « Le moulage. Pratiques historiques et regards contemporains » organisées par la Cité de l'architecture et du patrimoine et le musée du quai Branly, les 14 et 15 novembre 2012.

1 À l'occasion de la première journée d'étude consacrée aux moulages, organisée au musée du Louvre en 2011, j'avais expliqué en quoi le terme «moulage », utilisé pour désigner un objet, ne pouvait pas, à mon sens, s'appliquer aux plâtres du musée Rodin : les épreuves en plâtre (mais aussi en d'autres matières) que nous conservons sont bien tirées de moules, mais chacune doit être considérée comme une œuvre, faisant partie intégrante de la production d'un artiste, et non comme la réplique d'une œuvre qui serait seule " originale», notion délicate à manier dans le contexte de la sculpture. Seules les épreuves tirées de moules pris sur des marbres peuvent éventuellement être désignées comme «moulages de marbres ", par commodité. D'une manière générale, il me semble plus clair de réserver le terme « moulage » à la désignation d'un procédé technique, celui qui consiste à prendre une empreinte, à fabriquer un moule. Pour parler du résultat, de l'objet que l'on obtient par tirage ${ }^{1}$, je préfère employer le mot « épreuve », plus neutre et toujours plus juste au plan technique.

2 Ceci posé, nous pouvons aborder la question passionnante du rapport qu'Auguste Rodin (1840-1917) entretenait avec le moulage, procédé technique central dans ses pratiques de sculpteur. En ce domaine comme en d'autres, Rodin recourait aux services de collaborateurs spécialisés, et nous conservons le nom de plusieurs mouleurs ayant travaillé pour lui tout au long de sa carrière. Parmi eux, les plus importants sont les Guioché, Dieudonné le père et son fils Eugène, dont on peut, grâce aux archives conservées par le musée, retracer l'activité de 1897 à 1917. Alors que d'autres, plus tôt et 
plus tard dans la carrière de Rodin, n'ont travaillé que ponctuellement pour lui, les Guioché semblent avoir rapidement été des piliers de l'atelier du sculpteur - atelier au sens d'équipe, plus que de lieu, car ils eurent à exercer leurs talents en bien des endroits. Rodin leur confiait très régulièrement la tâche de mouler des œuvres en cours de création (modelages en terre, épreuves en plâtre retravaillées, éventuellement assemblées entre elles, et même des marbres en cours de pratique) afin de garder trace de leur forme et d'en tirer ensuite un ou plusieurs exemplaires.

\section{Rodin et ses mouleurs}

3 Voici quelques exemples concrets, tirés de lettres ${ }^{2}$ échangées entre l'artiste et ses collaborateurs, des diverses occasions d'utilisation du moulage tout au long du processus créatif :

[Début septembre 1909] Guioché, Prenez samedi matin à 9.50 le train à la gare Montparnasse pour Josselin. Vous vous arrêterez à Rennes, et vous prendrez le train omnibus pour la station de la Brohinière où vous changerez de train pour Josselin vous arrivez à $7 \frac{1}{2}$ et vous allez à l'hôtel de France. Vous apporterez du plâtre un quart de sac, pour mouler creux perdu la tête sans les épaules, la tête seule. Et emportez le creux avec vous à Paris. C'est mieux que de l'emballer dans la caisse. Cela ne sera pas lourd la terre glaise ôtée. Faites sans faute. Moi je pars étudier les cathédrales en Normandie et reviens.

4 C'est une véritable lettre de mission que Rodin donne ici à son mouleur: il lui faut se rendre en Bretagne, au château de Josselin, demeure du duc de Rohan, dont le sculpteur vient de modeler le portrait. Guioché devra en rapporter le moule à creux perdu, très précieux puisque seul témoignage de la terre modelée par l'artiste, disparue dans le processus. L'épreuve unique (le "plâtre original », au sens technique du terme) qui en sera tirée ultérieurement servira de point de départ à la suite du travail. On ne sait si Rodin avait la compétence pour réaliser lui-même un tel moule, mais il est évident qu'il gagnait beaucoup de temps en déléguant cette part du travail, ce qui lui donnait la liberté d'aller se promener pour admirer les cathédrales, l'une de ses grandes passions...

Fin juin 1907 : "Monsieur Guioché, Voulez-vous couler de suite un bourgeois de Calais $[. .$.

Prenez celui qui n'est pas fait - en staf ».

5 La demande semble toute simple, mais elle s'avère plutôt complexe quand on réalise qu'il s'agit de tirer une épreuve d'une figure grandeur nature, réalisée en plusieurs parties (le corps et les abattis). Le terme "en staf» désigne très probablement une technique qui semble avoir été une spécialité des Guioché, laquelle consiste à réaliser une épreuve d'épaisseur très fine et régulière, sans armature de métal ou de bois, mais parcourue d'un réseau orthogonal de renforts faits de cordons de filasse plâtrée.

17 mars 1911 : « Mon cher Guioché, Pourriez-vous me faire, je vous prie, une dizaine

de têtes, n'importe lesquelles qui aillent avec les figurines que vous estampez ».

Rodin a préalablement demandé à son mouleur de tirer par estampage (application de terre crue dans le creux du moule) plusieurs épreuves de diverses petites figures, qu'il envisage de retravailler; pour cela, il a besoin de têtes, elles-mêmes tirées de moules, mais Rodin se soucie peu de leur forme, pourvu qu'elles soient à la bonne échelle. Le sculpteur avait ainsi dans son répertoire de nombreux éléments anatomiques (têtes, torses, bras, mains, jambes, pieds...) modelés tout au long de sa carrière, qu'il remployait à loisir afin de gagner du temps. Parmi les têtes qui semblent avoir eu sa faveur (ou celle 
de son mouleur, puisqu'on voit par cette lettre que l'artiste se préoccupait à peine du choix du type), citons par exemple la Tête de la Femme slave, la Tête de Femme au chignon (qui existe en plusieurs tailles) ou encore celle dite de Malvina.

De Guioché à Rodin, 5 janvier 1915 : « L'estampage de la petite tête en marbre (Sirène) est faite dont je coule une épreuve. Je dois dire aussi que ce marbre n'est pas signé. Donc veuillez me faire savoir ce qu'il faut faire à ce sujet avant d'en donner livraison à Loïe Fuller qui doit venir le chercher ».

Il s'agit ici d'un marbre intitulé La Femme-Poisson, dit aussi Sirène, vendu à une collectionneuse californienne, Alma de Bretteville Spreckels, par l'intermédiaire de la danseuse américaine Loïe Fuller; il est conservé au California Palace of the Legion of Honour, prestigieux musée fondé par Mme Spreckels à San Francisco. Rodin développa dès les années 1890 l'habitude de faire mouler ses marbres avant de les céder, ce qui lui permettait d'en garder trace et d'en réaliser de nouvelles versions. En l'occurrence, ce marbre a fait l'objet d'une prise d'empreinte à la terre (estampage), et Rodin a pu, peu après, faire tailler un second exemplaire (Paris, musée Rodin) par l'un de ses praticiens, à partir d'une épreuve en plâtre tirée d'un moule à bon creux, certainement réalisé sur l'épreuve (a priori unique) tirée du moule en terre (fig. 1).

Figure 1

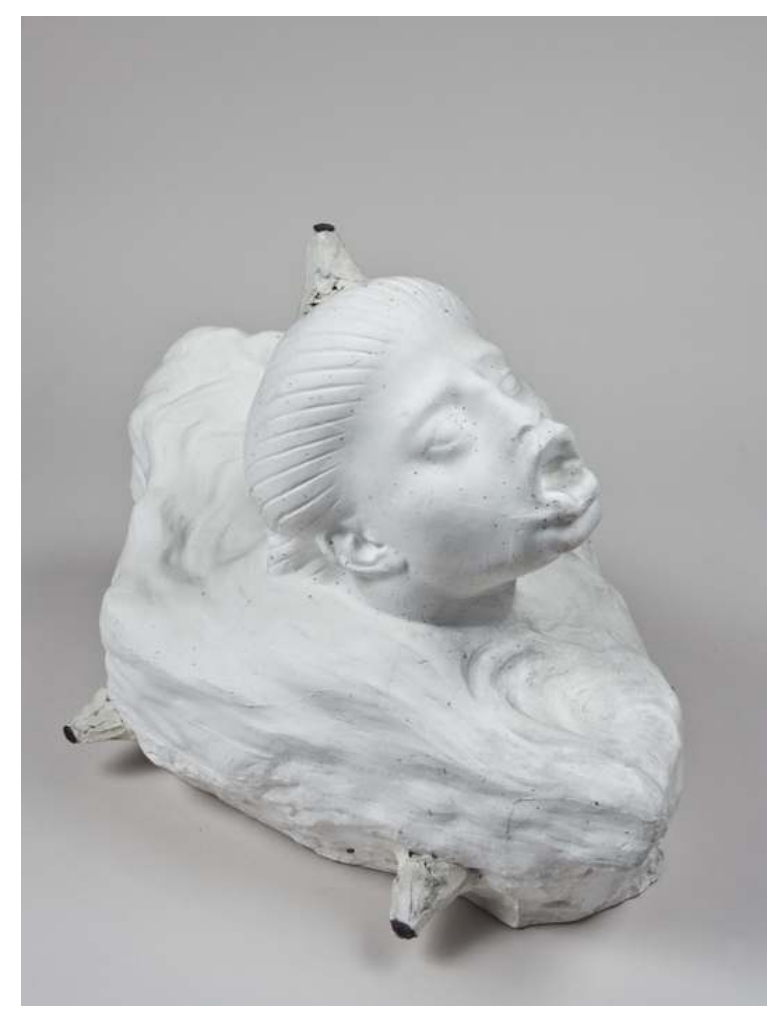

La Femme-Poisson, plâtre, moulage du premier marbre réutilisé comme modèle de mise aux points pour un second exemplaire, vers 1915. Paris, musée Rodin, S. 1877.

(c) Musée Rodin.

9 La compétence des Guioché était si reconnue que d'autres artistes souhaitaient en bénéficier, ce qui dit en creux combien Rodin s'était en quelque sorte attaché le monopole de leurs services. Une lettre de Jean-Paul Laurens en témoigne; elle fut adressée le 
20 septembre 1913 d'Yport (Seine-Maritime), où le peintre travaillait alors à une effigie sculptée de sa défunte épouse ${ }^{3}$ :

Mon cher ami,

Vous serait-il possible de me céder pour un jour ou deux le mouleur que vous employez en ce moment et dont $\mathrm{M}$. Roussel ${ }^{4} \mathrm{du}$ Trocadéro me dit le plus grand bien. J'ai exécuté ici, à la mémoire de ma chère disparue, un haut relief de figure tombale et, pour des raisons pratiques, j'aurais besoin d'en faire prendre l'empreinte entre le 24 et 30 septembre -2 jours suffiraient je crois pour ce travail.

Voyez mon cher ami si vous pouvez renoncer à Mr Guioché pour 2 jours et ditesmoi lesquels, afin que j'aie le temps de me retourner.

$\mathrm{Au}$ besoin, si cela vous était impossible ${ }^{5}$, j'aurais encore recours à vous pour me trouver quelqu'un à sa place.

Merci d'avance mon cher Rodin et croyez-moi votre affectueusement dévoué.

JP Laurens

Il s'agirait naturellement de faire venir le mouleur à Yport.

\section{Pratiques traditionnelles}

La prise d'empreinte et le tirage d'épreuves sont bien entendu des pratiques traditionnelles en sculpture, étroitement liées au travail de modelage dont elles permettent de garder trace et de démultiplier le résultat. Rodin y fut initié au cours de sa formation, à la fin des années 1850 , et eut très vite l'occasion d'en voir les avantages quand il travailla pour divers ateliers au début des années 1860 . On a coutume de dire qu'il apprit beaucoup au contact d'Albert-Ernest Carrier-Belleuse, dont il fut un proche collaborateur à partir de 1864 et jusqu'à 1871 (puis à nouveau vers 1878), et il est certain qu'il put y constater l'efficacité du partage des tâches et des techniques de création de modèles par déclinaison de motifs préexistants. Carrier-Belleuse avait tout particulièrement développé l'édition en terre cuite, et nous savons que Rodin pratiqua lui-aussi beaucoup ce matériau dans les années 1870 .

11 On imagine souvent trop vite qu'une œuvre en terre cuite est nécessairement le résultat d'un modelage, alors qu'il est bien plus fréquent qu'elle ait été créée par estampage dans un moule. L'épreuve en terre crue ainsi obtenue peut être facilement retouchée (coutures arasées, détails ravivés ou modifiés, éléments ajoutés...) et cuite. Par ce moyen, l'artiste peut obtenir rapidement et à moindre frais plusieurs exemplaires identiques, ou au contraire distincts, qui lui rapporteront plus d'argent que le modelage «original», disparu dans les opérations de moulage à creux perdu. Le musée Rodin conserve un bel exemple de cette pratique, un ensemble de trois petits groupes composés par assemblage d'épreuves tirées de moules pris sur deux figures, une jeune femme et un petit enfant. Chacun de ces groupes est unique, Rodin ayant pris soin de faire varier la position de l'enfant, devenu un Amour, par rapport à la figure féminine, intitulée Vénus (fig. 2). Nous savons qu'il créa ces groupes en 1871, lors de son séjour à Bruxelles, où il était venu rejoindre Carrier-Belleuse ${ }^{6}$. Ces œuvres sont en fait parmi les premières qu'il produisit après la rupture avec son patron, et qu'il vendit sous son nom, mais elles témoignent éloquemment de ce qu'il avait appris dans l'atelier qu'il venait de quitter. 
Figure 2

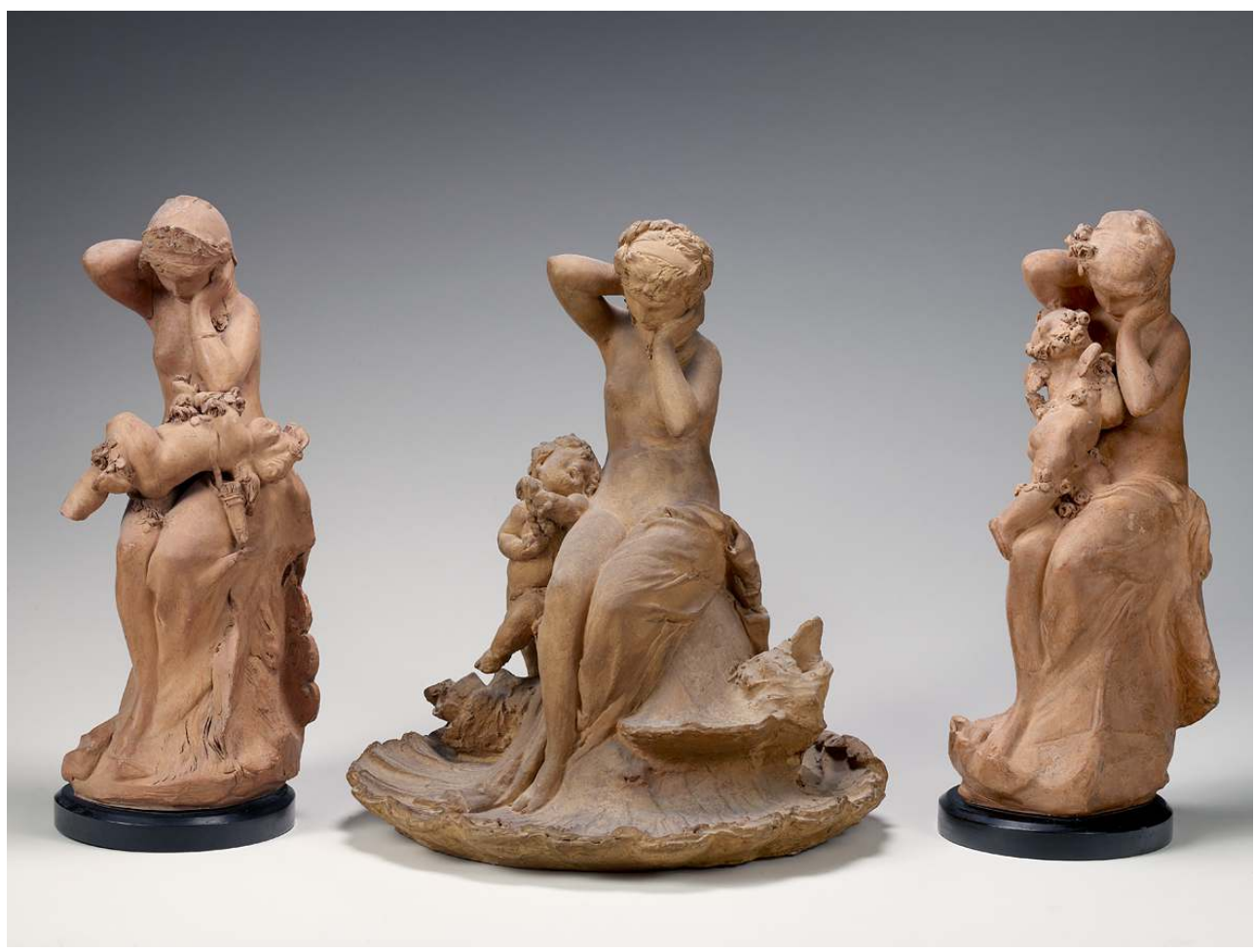

Secret d'amour, La Toilette de Vénus et Vénus et l'Amour, terre cuite, 1871 ; groupes composés par assemblage d'épreuves de chacune des deux figures. Paris, musée Rodin, S. 294, S. 1193 et S. 355.

(c) Musée Rodin.

Rodin affirma peu à peu son style personnel autour de 1880, sans rompre pour autant avec les pratiques traditionnelles, qu'il développa plutôt à son profit. L'un des grands avantages du moulage est de permettre le changement de matériau: de la terre crue au plâtre, puis du plâtre au bronze, à la terre, à la porcelaine, etc. Les exemples sont légion, mais j'en ai retenu quelques-uns qui illustrent les frontières très perméables entre sculpture et arts décoratifs - ou qui démontrent tout simplement que ces frontières ont peu de réalité, mais ceci est un autre débat ${ }^{7}$. Le premier est un petit bas-relief, La Sirène, créé sans doute vers 1885 pour servir de décor sur la façade de la villa de Maurice Fenaille, collectionneur et mécène de Rodin, à Neuilly. Le sculpteur modela cette œuvre en terre, puis il la fit mouler, couler en plâtre et traduire en pierre. Une épreuve en plâtre servit de modèle à une fonte en bronze que Fenaille plaça dans un cadre en bois, afin de l'accrocher au mur (offert par lui au musée des Arts décoratifs en 1908). Rodin poursuivit l'exploitation de ce motif, puisqu'il l'intégra à sa Porte de l'Enfer, au plus tard à la fin des années 1890. 
Figure 3

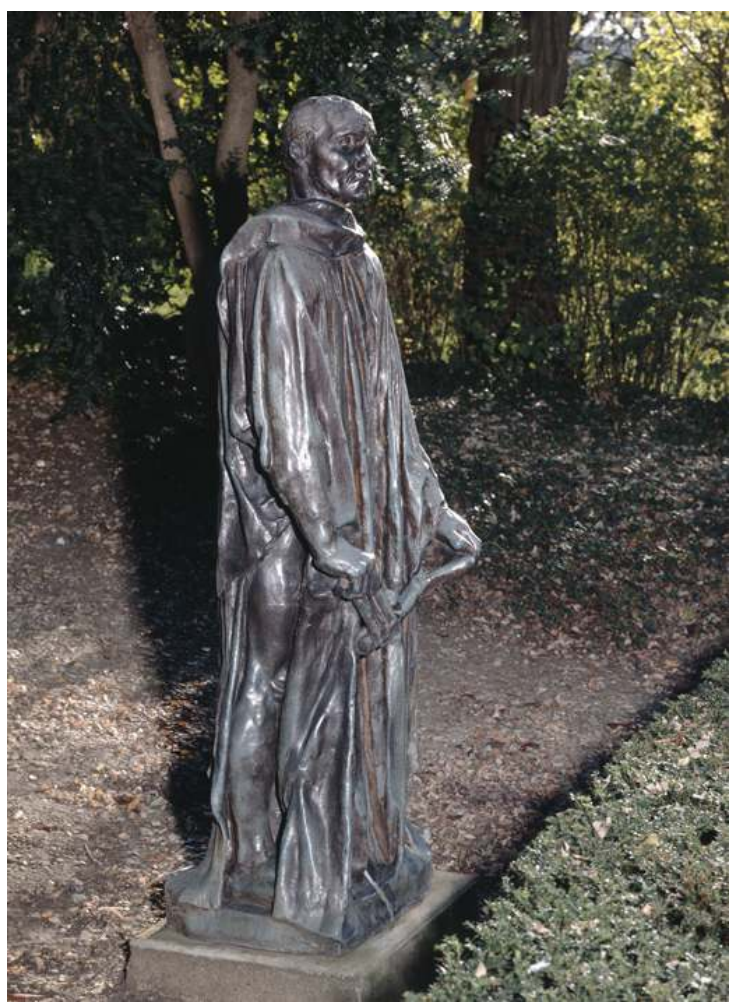

Jean d'Aire, grès émaillé exécuté par Paul Jeanneney, 1903-1904. Paris, musée Rodin, S. 152.

(c) Musée Rodin.

Dans la même logique, mais avec la collaboration de céramistes, Rodin fit exécuter en grès certaines de ses œuvres autour de 1900 : Rodin confia ainsi des modèles à Ernest Chaplet ${ }^{8}$, Edmond Lachenal $^{9}$ ou Paul Jeanneney. Ce dernier réalisa notamment plusieurs exemplaires du buste de l'un des Bourgeois de Calais, Jean d'Aire ${ }^{10}$, ainsi que deux épreuves de la figure complète ${ }^{11}$, grandeur nature, ce qui est un véritable tour de force technique (fig. 3) : elles sont composées de plusieurs éléments tirés séparément de leurs moules et assemblés, mais elles ont été séchées, cuites et émaillées comme des pièces d'un seul tenant. Enfin, Rodin fit traduire en pâte de verre certaines de ses œuvres, plutôt des visages féminins, par le spécialiste de ce matériau, Jean Cros. Cette technique relève bien $\mathrm{du}$ « moulage » puisqu'elle consiste à cuire dans un moule du verre réduit en poudre plus ou moins fine, en y ajoutant des pigments qui colorent localement la pâte. Le portrait de l'actrice japonaise Hanako, modelé vers 1906-1908, prend ainsi l'apparence étrange d'un visage aux couleurs proches des carnations réelles, mais figé dans une sorte de " catalepsie vitreuse ${ }^{12}$ » (fig. 4). 
Figure 4

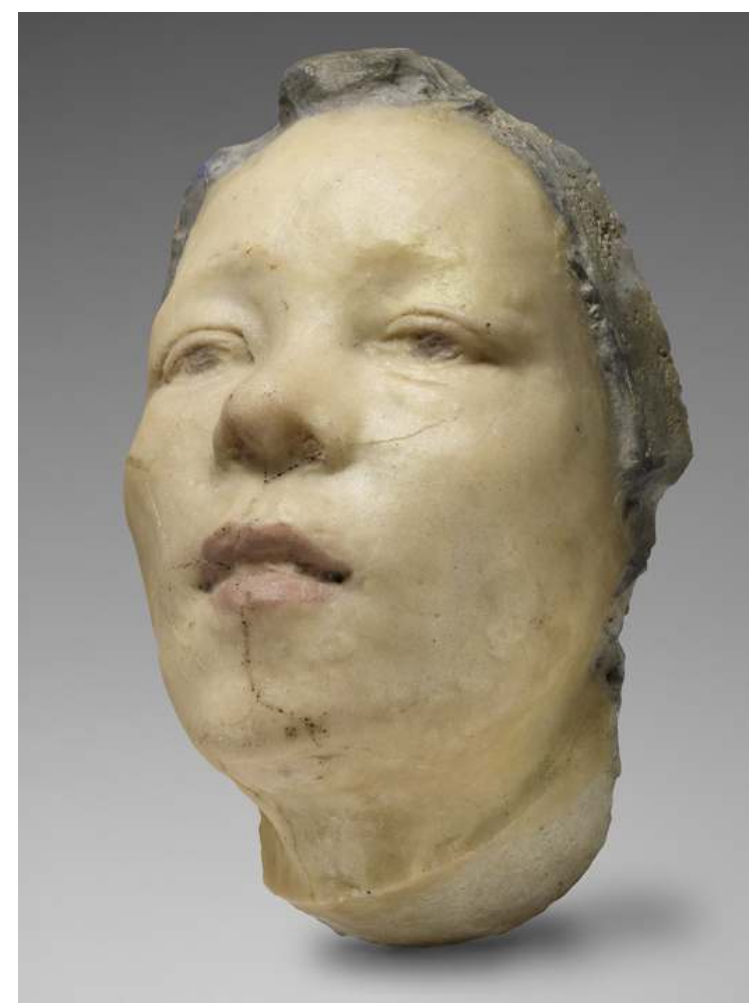

Hanako, masque type E, pâte de verre exécutée par Jean Cros, vers 1911. Paris, musée Rodin, S. 454.

(c) Musée Rodin.

Autre manière de changer de matériau, le passage du marbre au bronze est fréquemment pratiqué par Rodin. La lettre de Guioché à Rodin du 5 janvier 1915 (voir ci-dessus), évoquant « l'estampage de la petite tête en marbre (Sirène) », témoigne du fait que Rodin faisait mouler bon nombre de ses marbres avant de les livrer. Les épreuves en plâtre tirées des moules ainsi réalisés servaient bien souvent de modèles pour la fonte d'exemplaires en bronze, à l'encontre des discours convenus sur la nécessité de concevoir différemment une œuvre en vue du marbre et du bronze - discours tenu par Rodin luimême... Le cas extrême de cette pratique est certainement représenté par les éditions en bronze du Baiser, réalisées à partir d'un moulage du premier marbre de ce sujet (1889-1898, musée Rodin). Rodin avait cédé les droits de reproduction à la maison Barbedienne en 1898 (fig. 5), d'abord pour deux dimensions (70 et $25 \mathrm{~cm}$, tandis que le marbre mesure $180 \mathrm{~cm}$ de haut) puis pour deux autres (60 et $40 \mathrm{~cm}$ ). Pour chacune de ces quatre tailles, plusieurs dizaines d'exemplaires furent exécutés jusqu'à la fin du contrat en 1918, participant à la diffusion de cette œuvre et à son affirmation comme l'une des icônes de la sculpture mondiale ${ }^{13}$. 


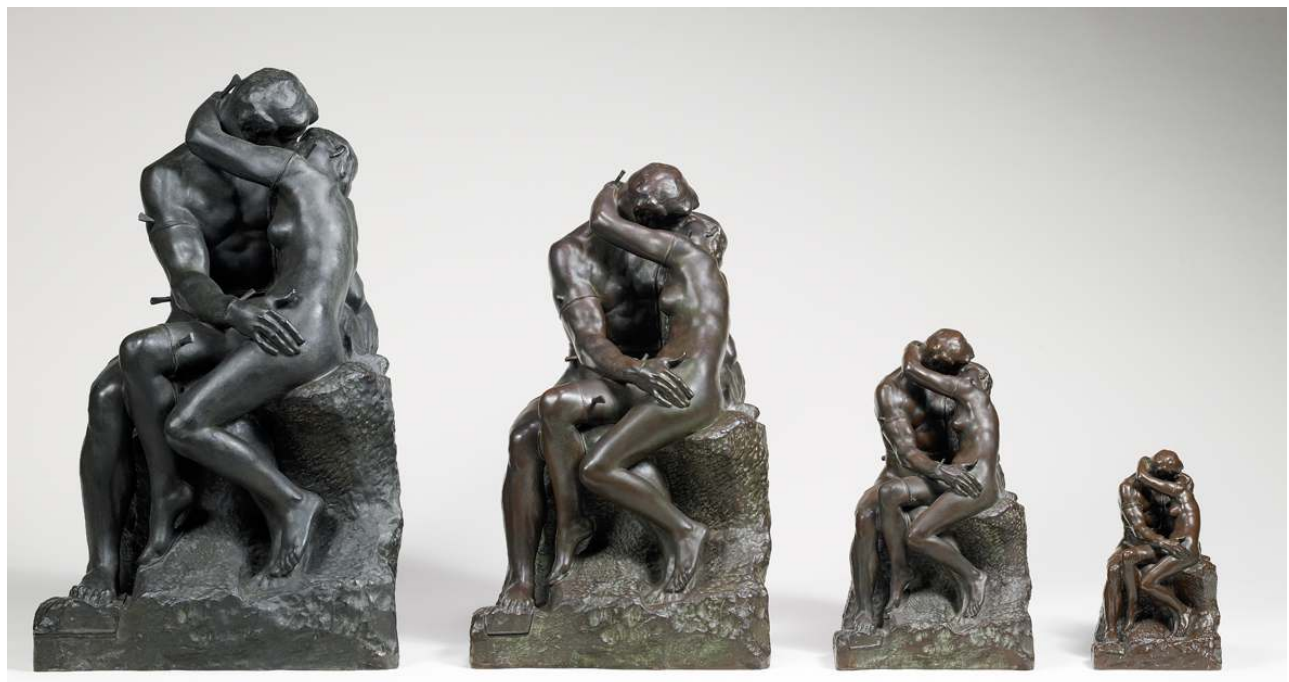

Le Baiser, bronze, chefs-modèles créés par la maison Barbedienne par réduction du marbre, en vue de l'édition en quatre tailles différentes, 1898-1904. Paris, musée Rodin, S. 2809, S. 2393, S. 2061 et S. 776.

(c) Musée Rodin.

\section{Développement d'usages plus propres à Rodin}

Au début des années 1880, alors qu'il abordait la quarantaine, Rodin affirma son style et sa position dans le milieu artistique, grâce notamment à la commande de la Porte de l'Enfer (16 août 1880). Ce projet était relativement modeste au départ, puisqu'il s'agissait simplement de proposer une maquette de porte ornée de bas-reliefs, pour le musée des Arts décoratifs que le gouvernement voulait créer à Paris. Pourtant, le sculpteur se mit au travail avec passion, et créa tout un monde de petites figures et de groupes destinés à venir peupler les vantaux de cette porte inspirée de L'Enfer de Dante. C'est dans ce contexte qu'il fut amené à pousser plus loin les techniques traditionnelles de composition par variation et déclinaison de motifs. Les Trois Ombres en constituent un parfait exemple : ce groupe destiné à couronner la Porte résulte de l'assemblage de trois exemplaires de l' Ombre (fig. 6) elle-même dérivée du grand Adam créé par Rodin en 1880-81. En disposant côte à côte ces trois épreuves tirées d'un même moule, alignées mais tournées chacune selon un angle différent, Rodin a composé une troublante image de l'abattement éprouvé par les âmes damnées, devenues anonymes dans la foule infernale. Leur caractère identique ne saute pas immédiatement aux yeux, c'est leur pose douloureuse que l'on remarque d'abord, mais on réalise peu à peu qu'il s'agit de la même figure, et le groupe n'en paraît alors que plus pathétique. 
Figure 6

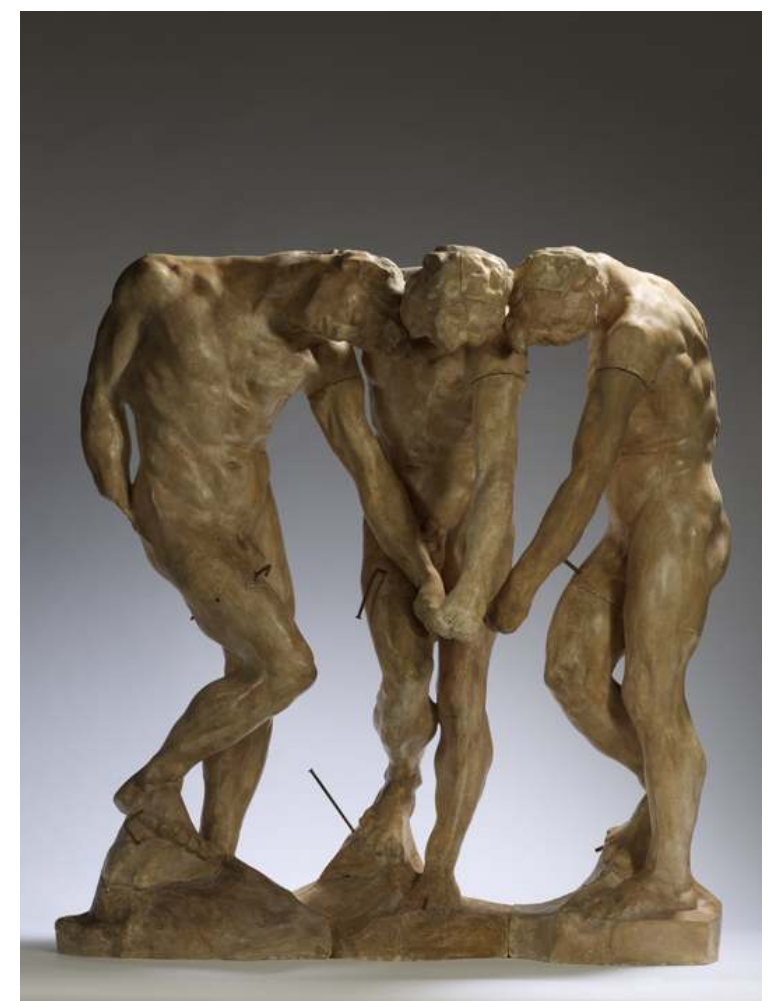

Les Trois Ombres, plâtre, vers 1885. Paris, musée Rodin, S. 3970.

(c) Musée Rodin.

Rodin fit de sa Porte à la fois un creuset où il mêla des figures et des groupes créés au fil des années, et un réservoir dont il tira régulièrement des éléments auxquels il donna une existence autonome. Il expérimenta avec de plus en plus d'audace et de liberté les possibilités créatives de l'assemblage, comme en témoigne le beau groupe intitulé Je suis belle (fig. 7) d'après le premier vers de La Beauté, l'un des poèmes des Fleurs du Mal, autre grande source d'inspiration pour la Porte de l'Enfer. Rodin réutilisa une épreuve de la Femme accroupie et une de L'Homme qui tombe, deux figures créées dès 1882-1883, pour composer un groupe nouveau : il modifia légèrement la figure féminine (base supprimée, bras droit tendu vers le sol) et celle de l'homme (jambes appuyées au sol, bras remontés et pliés) afin que ce dernier soulève sa compagne. L'expression de chaque figure se trouve transformée par leur simple juxtaposition : la pose de l'homme, dos arqué, tête rejetée en arrière, suggère dorénavant l'effort plus que la souffrance, tandis que celle de la femme prend une nuance d'abandon qu'elle n'avait pas au départ. 
Figure 7

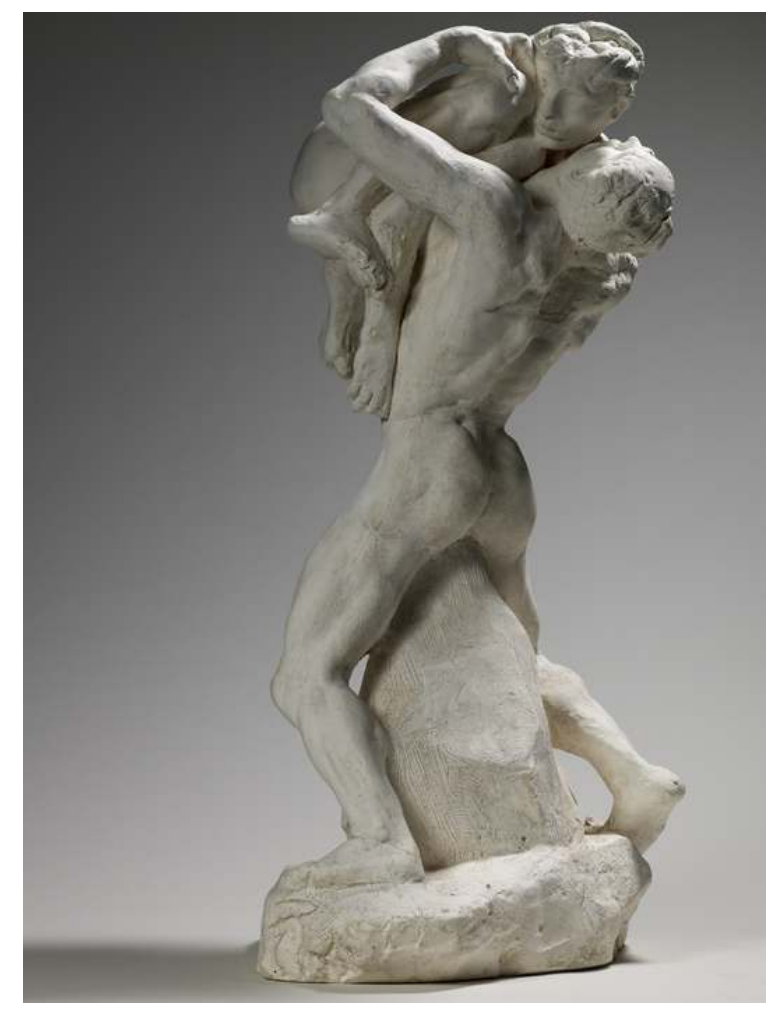

Je suis belle, plâtre, vers 1885. Paris, musée Rodin, S. 219.

(C) Musée Rodin.

Cette pratique, dans laquelle les procédés de moulage et de tirage tiennent une place centrale, devint rapidement une caractéristique de l'art de Rodin. On peut ainsi suivre au fil des années, voire des décennies, l'évolution d'un motif et sa déclinaison au gré de multiples variations et combinaisons. Le personnage masculin du groupe Fugit Amor (fig. 8), par exemple, devient L'Enfant prodigue lorsqu'il est isolé et appuyé en position verticale sur un tertre dont il semble devoir tomber. Réduit à l'état de torse, il devient une sorte d'anse sur un vase antique appartenant aux collections personnelles du sculpteur (fig. 9), en pendant avec un autre torse qui lui est proche, celui de l'Adolescent désespéré. Ce dernier forme avec une figure féminine appelée L'Aube un groupe sans titre particulier, l'un de ces très nombreux assemblages créés par Rodin dans le secret de son atelier, pour son plaisir, dont certains ont donné lieu à des œuvres plus abouties tandis que d'autres sont restés en l'état. Et ainsi de suite, chaque figure prenant sa place dans un gigantesque arbre généalogique dont les branches et les générations sont liées par des étapes de moulage et de tirage d'épreuves, lesquelles sont retouchées, modifiées, assemblées, moulées à nouveau, etc. 
Figure 8

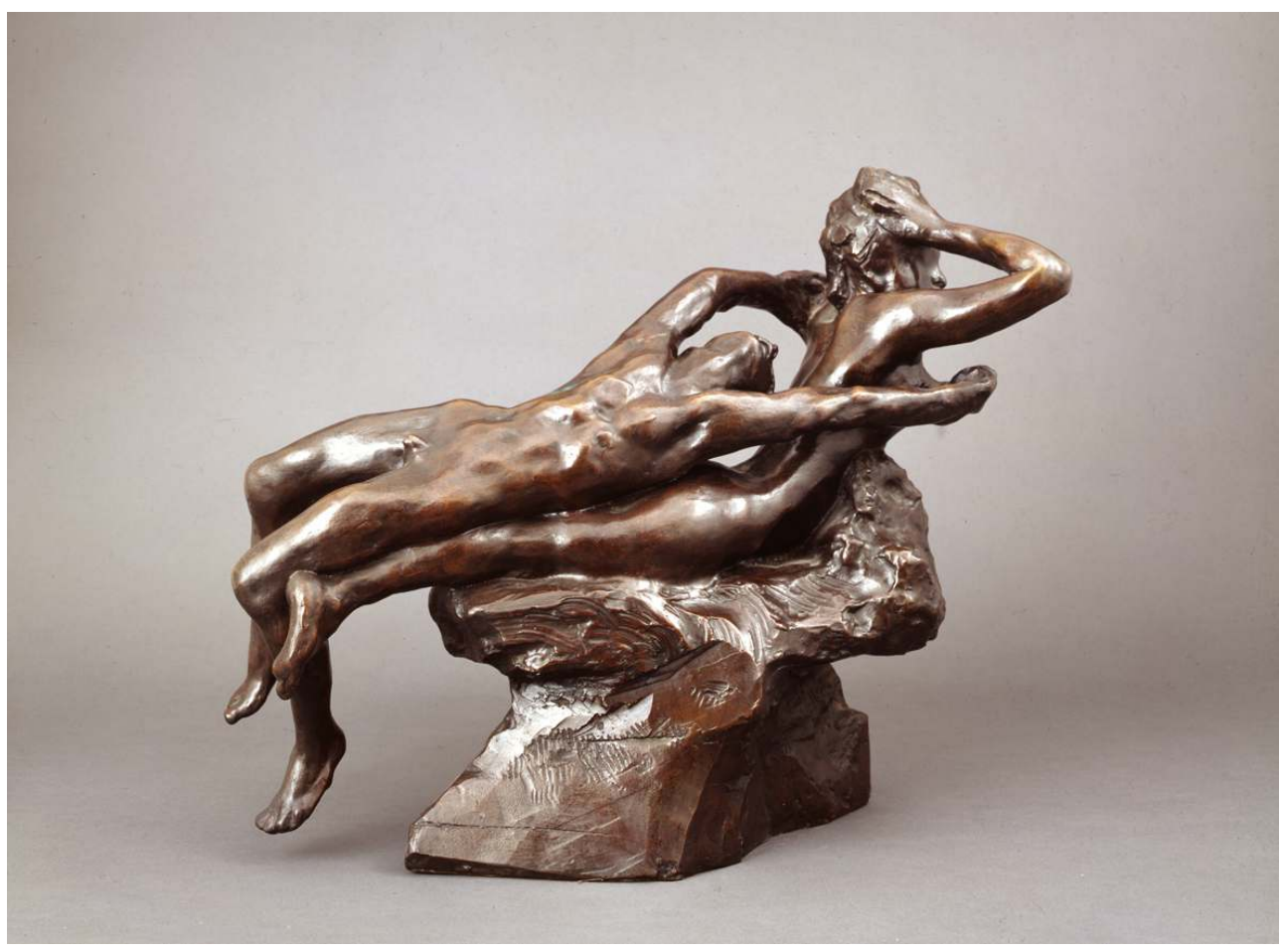

Fugit Amor, bronze, vers 1885. Paris, musée Rodin, S. 598.

(c) Musée Rodin. 
Figure 9

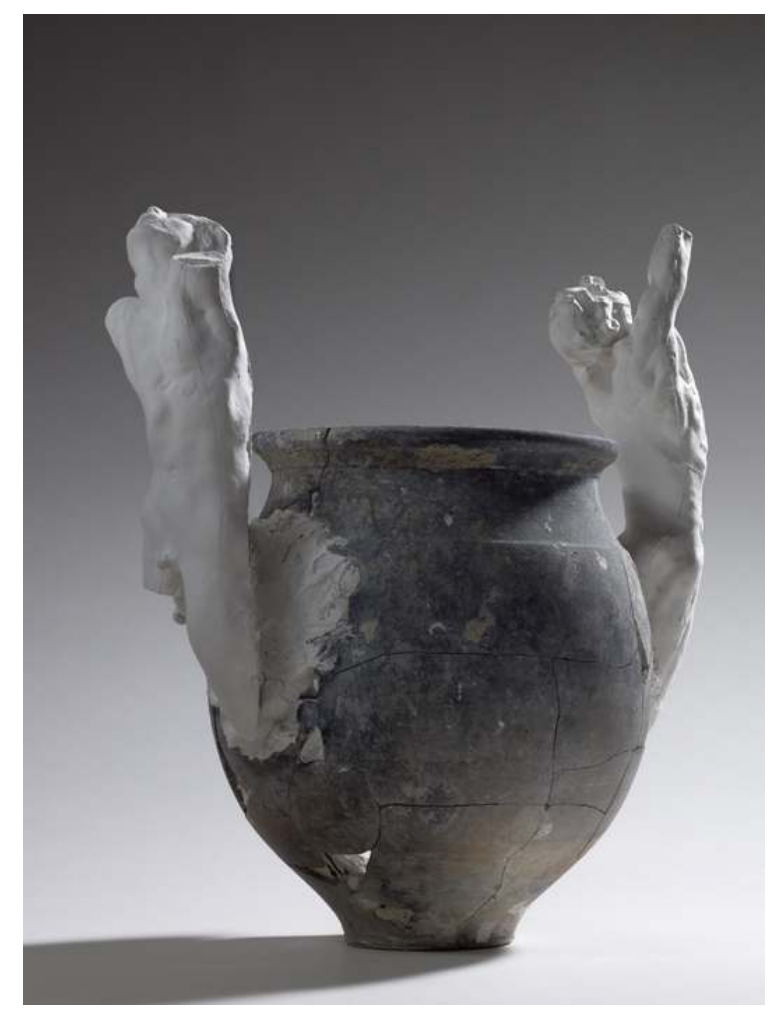

Assemblage : vase antique et torses de l'Enfant prodigue et de l'Adolescent désespéré, céramique et plâtre, vers 1900-1910. Paris, musée Rodin, S. 3614.

(c) Musée Rodin.

\section{Pratiques inédites}

Poussant ses recherches plus loin que ses contemporains, Rodin expérimenta des techniques inédites, du moins à notre connaissance. Dans le domaine du portrait, par exemple, lorsque le résultat de son modelage ne lui convenait pas - ou quand le modèle se montrait aussi exigeant que peu satisfait - Rodin gagnait du temps en évitant de repartir à zéro : il faisait mouler son modelage et tirer une ou plusieurs épreuves en terre crue qu'il pouvait retoucher. Autre possibilité : faire tirer une épreuve en plâtre, et modifier ses détails, voire ses volumes, à l'aide de pâte à modeler, communément appelée plastiline ${ }^{14}$. La série des études pour le buste de Clemenceau, constituée d'une trentaine d'états en terre cuite et en plâtre (fig. 10), est de loin le plus beau cas que nous connaissions dans ce genre $^{15}$. Rodin y travailla entre 1911 et 1913, peinant à aboutir à une effigie qui plaise à son modèle. Le 8 juillet 1911, il écrivit à son secrétaire Mario Meunier : « Mon cher Mario, allez voir à Meudon Guioché et dites-lui que M. Clemenceau vient poser lundi à 10 heures. Il faut que j'ai mon buste estampé et porté chez moi à $9 \mathrm{~h}$ rue de Varenne. Il faut qu'il soit prêt plutôt demain dimanche pour plus de sûreté ». 
Figure 10

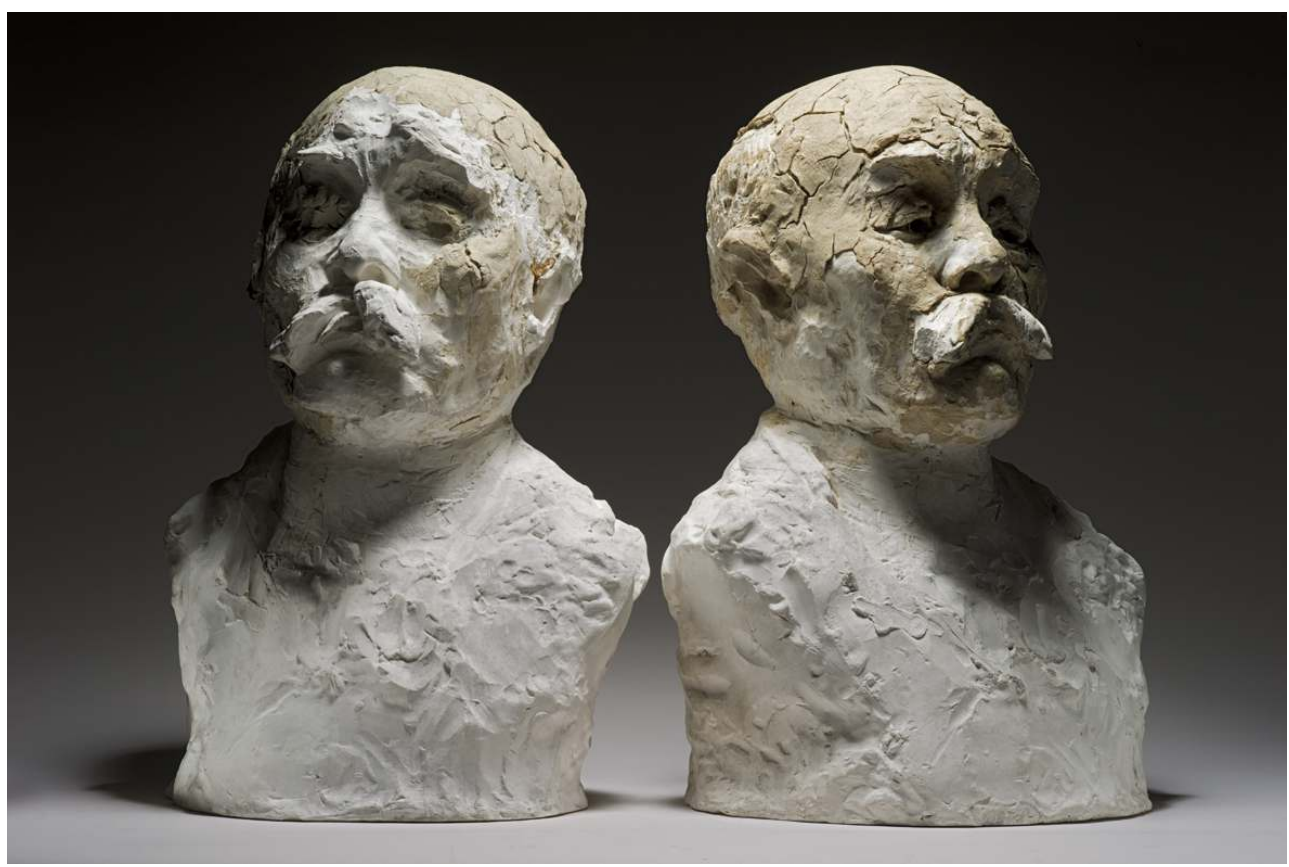

Clemenceau, plâtre retouché à la plastiline, vers 1912. Paris, musée Rodin, S. 1982 et S. 1730.

(c) Musée Rodin.

Plusieurs terres sont conservées, dans lesquelles les détails varient ; d'autres ont disparu, car elles ont été moulées à creux perdu et remplacées par des plâtres, qui eux-mêmes donnèrent lieu à de nouvelles évolutions. Interrogé par un journaliste, Rodin expliqua en 1912 que chaque buste « n'est qu'une étape vers le Clemenceau idéal et parfait auquel je pense. C'est, pour moi, quelque-chose comme un état d'eau-forte... Plus tard, j'y reviendrai, je corrigerai, je retoucherai... J'ai de mon modèle une image très nette $»^{16}$. La comparaison avec les pratiques d'un graveur est tout à fait pertinente, et nous amène à considérer de manière nouvelle la place des opérations de moulage et de tirage : certes, en sculpture, l'artiste travaille en positif sur des objets successifs, et non en négatif sur le même support (la plaque), mais le fait de multiplier les épreuves et de faire évoluer la forme de l'une à l'autre me semble être une approche jamais documentée avant Rodin.

Le sculpteur innova également dans le domaine des marbres, qui paraît pourtant le moins propice à l'utilisation des techniques de moulage. Au cours $d u x^{e}$ siècle, on a trop longtemps reproché à Rodin de n'avoir pas taillé lui-même ses marbres, certains commentateurs considérant même que ces œuvres ne pouvaient être pleinement considérées comme étant siennes, puisqu'elles n'étaient en quelque sorte pas de sa main. Il faut renverser la perspective, et bien noter que chez Rodin la phase de conception ne se réalisait que partiellement dans le modèle qu'il confiait à son praticien. Ce dernier recevait en effet, avec l'objet, des consignes par lesquelles le maître indiquait comment chaque partie devait être traitée. Rodin passait ensuite régulièrement pour voir le travail en cours, et correspondait aussi par lettre avec ses praticiens, si bien que nous avons de nombreux témoignages de leurs relations quotidiennes et des changements de parti que l'artiste introduisait en cours de pratique. Il y a donc, à mesure que l'on avance dans la carrière, une distance de plus en plus grande entre l'aspect du modèle et celui du marbre (fig. 11, fig. 12), lequel doit pourtant bien être considéré comme l'œuvre définitive. 
Certes, les maquettes, qui sont souvent réalisées par assemblage d'éléments tirés de moules, nous fascinent parce qu'on y lit directement le travail personnel de l'artiste, mais Rodin ne les a que rarement exposées.

\section{Figure 11}

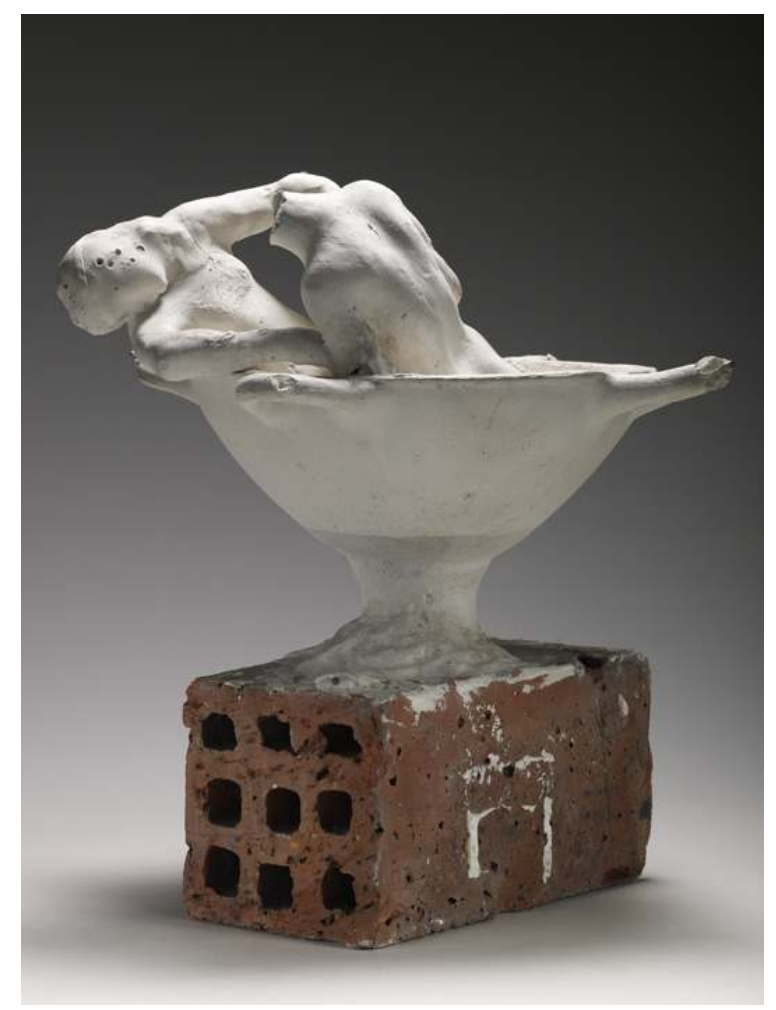

Fleurs dans un vase, plâtre, modèle de mise aux points, vers 1905. Paris, musée Rodin, S. 3700.

(C) Musée Rodin. 
Figure 12

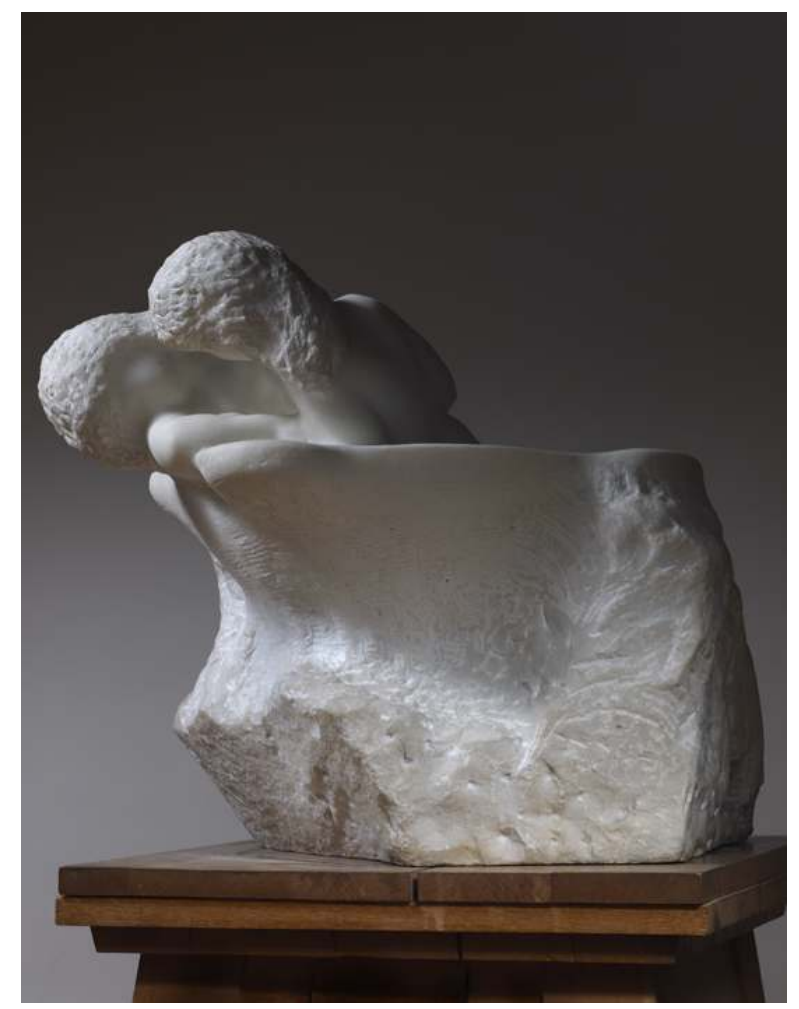

Fleurs dans un vase, marbre, vers 1905. Paris, musée Rodin, S. 1418.

(C) Musée Rodin.

Les choses ne s'arrêtent pas là : comme nous l'avons vu dans la première partie, Rodin faisait fréquemment mouler ses marbres, et n'hésitait pas à utiliser une épreuve tirée du moule pris sur la pierre pour faire tirer des bronzes. Mais ce moulage pouvait aussi servir de modèle de mise aux points pour l'exécution d'une nouvelle version en marbre ; celle-ci pouvait être identique à la précédente ou au contraire donner lieu à une évolution plastique, notamment dans le rapport entre la partie figurative et le reste du bloc, laissé de plus en plus souvent dans un état brut. Dans certains cas extrêmes, un moulage de marbre peut avoir été utilisé comme point de départ pour une nouvelle composition, ellemême traduite en marbre ensuite. C'est le cas d'une épreuve tirée d'un moule pris sur un marbre actuellement non localisé, intitulé L'Éveil, à laquelle Rodin a adjoint une épreuve en plâtre de son Athlète américain ; après avoir été retouché à la pâte à modeler (bras de l' Éveil, jambes de l'Athlète...), le groupe fut confié à un praticien (fig. 13). Dans le marbre qui résulte de cette opération, nul ne peut lire le rôle joué par le moulage du marbre qui a servi de point de départ à cette composition (fig. 14). 
Figure 13

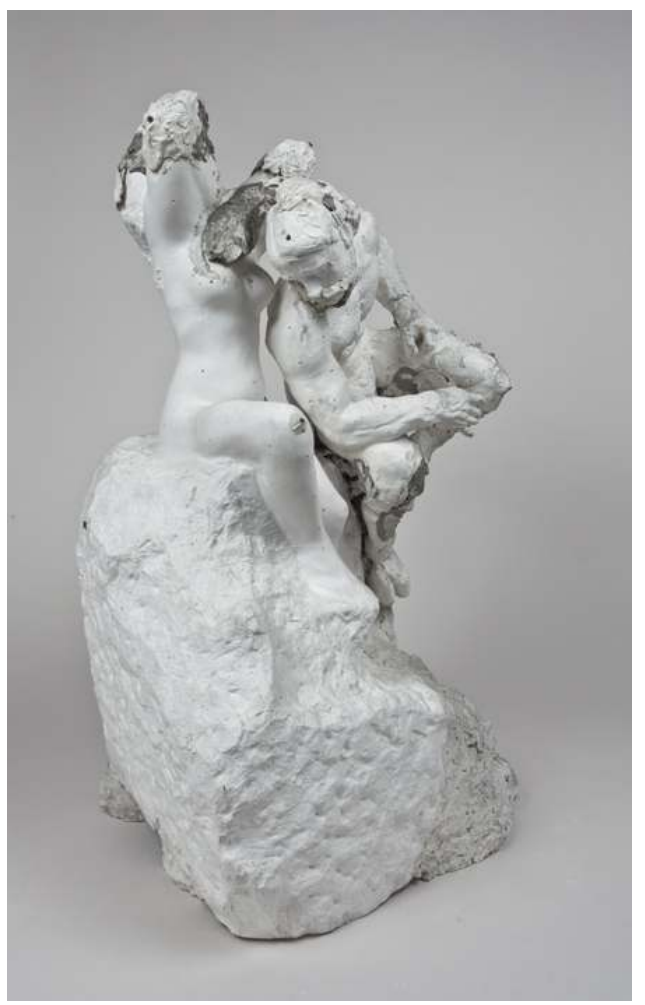

Assemblage d'un moulage du marbre L'Éveil et d'une épreuve de l'Athlète américain, plâtre, modèle de mise aux points pour Pan et Nymphe, vers 1910. Paris, musée Rodin, S. 2148.

(c) Musée Rodin. 
Figure 14

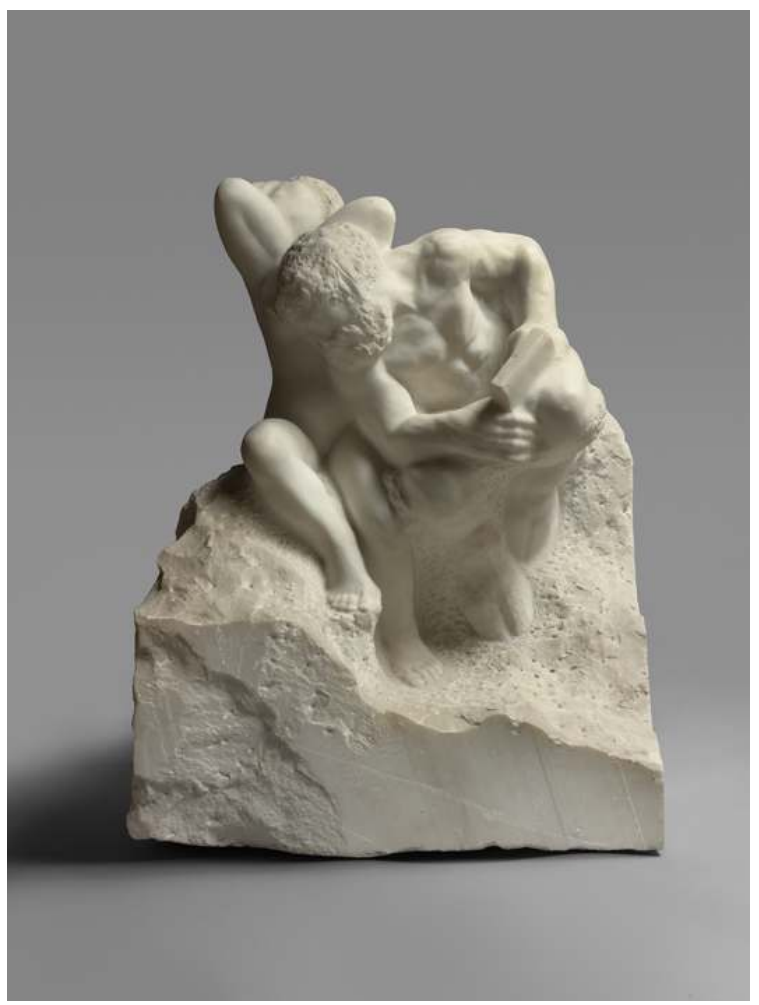

Pan et Nymphe, marbre, vers 1910. Paris, musée Rodin, S. 1205.

(c) Musée Rodin.

\section{Biographie}

Spécialiste de l'art français du XIX ${ }^{\mathrm{e}}$ siècle, François Blanchetière a mené ses premières recherches en histoire de l'art sur la formation et les débuts de Pierre Puvis de Chavannes. Il est l'un des conservateurs du musée Rodin depuis 2005, et a été commissaire de plusieurs expositions, notamment « Rodin, le Rêve japonais » (2007, avec B. Garnier) et « Corps et décors, Rodin et les arts décoratifs » (2009-2010). Il s'intéresse particulièrement aux aspects techniques de la sculpture : le moulage, mais aussi la fonte, la taille des marbres et les procédés d'agrandissement.

\section{BIBLIOGRAPHIE}

CASCIO, Agnès, et LEVY, Juliette. « Généalogie d'un portrait : Clemenceau par Rodin », Patrimoines $, \mathrm{n}^{\circ} 9,2013$.

LE NORMAND-ROMAIN, Antoinette. Rodin et le bronze. Catalogue des cuvres conservées au musée Rodin. Paris : Éditions du musée Rodin et RMN, 2007.

\section{Catalogues d'exposition :}


LE NORMAND-ROMAIN, Antoinette, JUDRIN, Claude, VASSALO, Isabelle. Vers L'Âge d'Airain. Rodin en Belgique (cat. exp. Paris, musée Rodin, 18 mars-15 juin 1997). Paris : musée Rodin, 1997.

BLANCHETIÈRE, François, BOUILLON, Jean-Paul, DE DECKER, Aurélie et al. Corps et décors. Rodin et les arts décoratifs (cat. exp. Évian, palais Lumière, 2009, et Paris, musée Rodin, 2010). Paris : Alternatives, 2009.

\section{NOTES}

1. - De la même manière, le mot «tirage » me semble devoir être réservé au procédé technique par lequel on tire une épreuve d'un moule.

2. - Toutes conservées aux archives du musée Rodin, comme les autres lettres citées par la suite. 3. - Plâtre (original ?) au musée des Augustins, Toulouse ; bronze, fonte Hohwiller, 1914, église de Fourquevaux (Haute-Garonne) ; une autre épreuve en bronze, fonte Alexis Rudier (avant 1925), au musée d'Orsay.

4. - Jules Roussel, conservateur adjoint du musée de Sculpture comparée.

5. - Il semble que Guioché n'ait finalement pas pu faire le voyage.

6. - Voir LE NORMAND-ROMAIN, Antoinette, JUDRIN, Claude, VASSALO, Isabelle. Vers L'Âge d'Airain. Rodin en Belgique (cat. exp. Paris, musée Rodin, 18 mars-15 juin 1997). Paris : musée Rodin, 1997.

7. - Pour ce qui est de Rodin, voir BLANCHETIÈRE, François, BOUILLON, Jean-Paul, DE DECKER, Aurélie et al. Corps et décors. Rodin et les arts décoratifs (cat. exp. Évian, palais Lumière, 2009, et Paris, musée Rodin, 2010). Paris : Alternatives, 2009.

8. - Pour une Bacchante ou Faunesse assise riant en porcelaine émaillée ; op. cit., $\mathrm{n}^{\circ} 64$.

9. - Pour plusieurs masques de Pleureuse en grès émaillé ; op. cit., n 145 et 146.

10. - Voir op. cit., n 149 et 150.

11. - Voir op. cit., $\mathrm{n}^{\circ}$ 148. Outre l'exemplaire du musée Rodin, un seul autre existe, au musée d'Art moderne et contemporain de Strasbourg (don des neveux de Jeanneney, 1921).

12. - Voir op. cit., $\mathrm{n}^{\circ} 151$ et 152.

13. - Voir LE NORMAND-ROMAIN, Antoinette. Rodin et le bronze. Catalogue des œuvres conservées au musée Rodin. Paris : Éditions du musée Rodin et RMN, 2007, p. 160-162.

14. - CASCIO, Agnès, et LEVY, Juliette. "Généalogie d'un portrait: Clemenceau par Rodin», Patrimoines, $\mathrm{n}^{\circ}$ 9, 2013, p. 80-89.

15. - Ce n'est pas le seul : plusieurs bustes dont le musée conserve des études ont fait l'objet d'un travail de ce type (notamment Harriman et Hanako-Beethoven).

16. - Propos rapportés dans CHENNEVAL, Claude. « Pourquoi Rodin n'expose pas à la Nationale ». Excelsior, $\mathrm{n}^{\circ}$ 516, 14 avril 1912, p. 3.

\section{RÉSUMÉS}

Dans le contexte de l'atelier d'un artiste, le terme de moulage doit être réservé à la désignation de l'opération qui consiste à fabriquer un moule; l'objet que l'on en tire est une épreuve, étape ou résultat final dans le processus de création d'une œuvre. Partant des pratiques de moulage et de tirage d'épreuve traditionnellement utilisées en sculpture, Rodin a développé, avec l'aide de 
ses mouleurs, des usages nouveaux, faisant preuve d'une grande liberté (multiplication des exemplaires et des variantes, changement de matériau, assemblage d'épreuves diverses...).

In the context of an artist's studio, the term 'moulding' (moulage) should be limited to the operation which involves making a mould. The object produced from this mould is a 'proof' or 'test' (épreuve), a stage, or the result in a process of creating a work of art. Using the moulding and proof casting techniques traditionally employed by sculptors, Rodin with the help of his moulders, developed a range of new practices, showing great freedom of inspiration: multiplying copies and variations, using different materials, assembling different test casts...

\section{INDEX}

Mots-clés : assemblage, bronze, épreuve, estampage, grès, Guioché, marbre, moulage, pâte de verre, plâtre, Rodin, Roussel, terre crue, terre cuite, tirage, Trocadéro

Keywords : assembly, proof, stamping, sandstone, guilloché, marble, moulding, cast, glass paste, plaster, raw clay, terra cotta, edition

\section{AUTEUR}

\section{FRANÇOIS BLANCHETIÈRE}

Conservateur du patrimoine, adjoint au responsable du service de la conservation du musée Rodin blanchetiere@musee-rodin.fr 